\title{
Stabilité des massifs rocheux fissurés
}

\section{A. ASROUN}

Laboratoire de matériaux et hydrologie

Département de génie civil

Université

de Sidi Bel-Abbès BP 89

22000 Sidi Bel-Abbès, Algérie

a_asroun@yahoo.fr

\section{J.-L. DURVILLE}

Laboratoire central des ponts et chaussées

58, bd Lefebvre 75732 Paris Cedex 15

\begin{abstract}
Dans cet article nous étudions les discontinuités dans les massifs rocheux fissurés : leurs origines, leurs caractéristiques géométriques et mécaniques, leur comportement mécanique, ainsi que différentes méthodes de renforcement des massifs rocheux fissurés notamment par ancrages passifs. Les résultats d'une étude expérimentale, réalisée au Laboratoire central des ponts et chaussées de Paris sur une discontinuité artificielle armée par une barre d'acier scellée sur toute sa longueur (ancrage passif), en faisant varier différents paramètres (résistance à la compression de la roche, inclinaison de la barre), sont présentés.
\end{abstract}

Mots-clés : discontinuités, massif rocheux, ancrage passif, renforcement.

\section{Stability of discontinuous rock masses}

rock masses : their origin, their geometrical characteristics, and the different methods of reinforcement of cracked rock masses especially with passive anchorage. The results of ar

experimental study realised at the Laboratoire central des Ponts et chaussées de Paris on an artificial discontinuity are presented.

Key words : discontinuities, rock masses, passive anchorage, reinforcement. 


\section{Introduction}

La résistance mécanique des roches intactes, déterminée sur des éprouvettes en laboratoire, est en général élevée; un tel matériau, s'il était homogène et continu, pourrait supporter des sollicitations plus importantes que celles auxquelles il est habituellement soumis, mais, à l'échelle des ouvrages de génie civil, la plupart des massifs rocheux se comportent comme des milieux discontinus, hétérogènes et anisotropes. Cette complexité du comportement des milieux rocheux est due à leur fracturation qui intervient à des échelles très variées. Les contraintes transmises par les fondations des ouvrages et les talus rocheux sont faibles et provoquent rarement la rupture de la roche intacte, donc, les ruptures dans les massifs rocheux se produisent essentiellement par ouverture des discontinuités préexistantes et glissement relatif des blocs ou des bancs. Par conséquent, la stabilité de ces massifs rocheux est gouvernée par les discontinuités géologiques préexistantes.

\section{2}

\section{Les discontinuités dans les massifs rocheux}

\section{1}

\section{Leurs origines}

On distingue dans la nature deux grands types de surfaces de discontinuités rocheuses d'origines différentes.

\section{2ifat}

\section{Les surfaces de discontinuités d'origine tectonique}

Ce sont des déformations mécaniques appartenant à trois grandes classes différentes :

1) Les plissements, dans lesquelles interviennent des phénomènes de plasticité et le temps. Ce sont des déformations relativement continues.

2) Les fractures, correspondent à des déformations discontinues et dans lesquelles interviennent des phénomènes de rupture.

3) La schistosité, qui affecte la structure de la roche elle-même.

\subsection{2.}

\section{Les joints de stratifications}

Les roches sédimentaires telles que marnes, calcaires, pélites, conglomérats, etc., présentent, généralement, une anisotropie liée à leur dépôt. Cette anisotropie originelle et affectant donc la matrice rocheuse est souvent soulignée par des plans de stratification qui marquent un changement ou un arrêt de sédimentation, ils séparent deux strates successives. Les plans de stratification présentent une grande continuité spatiale dans les dépôts marins sédimentés sur de vastes super- ficies et, au contraire, de brusques variations d'orientation pour certains faciès continentaux déposés de façon anarchique. L'échelle d'alternance est très diverse : de millimétrique pour des dépôts argileux, elle peut atteindre plusieurs mètres pour certains grès ou calcaires massifs.

\section{2}

\section{Leurs caractéristiques géométriques et mécaniques}

Le massif rocheux présente des surfaces de discontinuités découpant la masse rocheuse en une série de blocs plus ou moins imbriqués et reliés entre eux par la cohésion de leurs discontinuités, La résistance d'ensemble du massif est davantage conditionnée par les caractéristiques géométriques et les propriétés mécaniques de ces surfaces de discontinuités que par la résistance de la matrice rocheuse.

Du point de vue mécanique, ces discontinuités n'assurent pas la continuité dés déformations tangentielles. L'intensité de la fracturation conditionne les propriétés mécaniques et hydrauliques du massif Benjelloun et al. (1989), sa connaissance est indispensable à l'élaboration des procédés de traitement (injection, drainage, renforcement par barre, etc.)

Les dispositions des surfaces de discontinuité dans l'espace ainsi que leurs propriétés mécaniques sont déterminantes pour l'analyse de tous les problèmes de stabilité (appui de barrage, soutènements, fondations diverses, travaux souterrains, etc.)

\section{Les caractéristiques géométriques des discontinuités}

a) L'orientation. A l'échelle des ouvrages de génie civil les discontinuités sont assimilées à des plans, donc, pendage et azimut suffiront à préciser leur orientation. La projection stéréographique est la méthode de représentation des orientations des plans la plus utilisée. En général, les discontinuités sur un site se regroupent en un certain nombre $(1,2,3 \ldots)$ de familles définies par leur orientation.

b) La position et l'espacement. En général, la position absolue d'une discontinuité a moins d'importance que sa position relative par rapport aux discontinuités voisines qui est donnée par l'espacement entre discontinuités. Pour chaque famille de discontinuitès, on définit un espacement moyen égal à la moyenne des espacements.

c) L'ouverture. C'est la distance qui sépare les deux surfaces d'une discontinuité. Le tableau suivant donne une classification des ouvertures des discontinuités dans les roches.

d) La persistance. Il est difficile de cerner cette caractéristique en raison des dimensions limitées de la surface d'observation. D'un point de vue pratique, on utilise le rapport $R=1 / L$ entre la plus grande longueur observable à l'affleurement sur la même surface de discontinuité (l) et la plus grande dimension de l'affleurement dans cette direction (L). Ce rapport est compris entre 0 et 1 et permet de chiffrer l'importance de la discontinuité par rapport à l'affleurement. 
Classification of discontinuities openings.

\begin{tabular}{cc}
\hline Ouverture $\left(10^{-3} \mathrm{~m}\right)$ & Description \\
\hline$<0,1$ & Très fermée \\
$0,1-0,25$ & Fermée \\
$0,25-0,5$ & Partiellement ouverte \\
$0,5-2,5$ & Ouverte \\
$2,5-10$ & Modérément large ouverture \\
$>10$ & Large ouverture
\end{tabular}

\subsection{2.}

\section{Les caractéristiques mécaniques des discontinuités}

Les propriétés mécaniques les plus importantes des discontinuités dans les roches sont celles qui résultent de leur comportement au cisaillement : angle de frottement, cohésion, dilatance, rugosité, etc. (Bjurstrom, 1974; Bandis et al., 1981).

Leur résistance à des sollicitations de traction normales à leur surface est généralement très faible et souvent nulle. Elle est liée soit à l'existence des ponts de matière, soit à une cohésion induite par le remplissage.

\section{3}

\section{La stabilité des massifs rocheux fissurés}

Le massif rocheux affecté d'un réseau plus ou moins dense de fractures est composé de blocs formant un édifice généralement stable dans les conditions naturelles, mais dont l'équilibre peut être rompu, instantanément ou à terme, par les travaux d'exploitation du site.

L'analyse des conditions de stabilité commence par une description et une représentation des surfaces de discontinuités et se poursuit par l'analyse des conséquences géométriques : création des prismes ou d'autre polyèdres par intersection des familles de fractures (analyse cinématique) et s'achève par l'analyse des possibilités de rupture sur les surfaces limites de ces volumes (analyse mécanique).

\section{Le renforcement des massifs rocheux fissurés}

La complexité des problèmes des talus rocheux et le nombre des parametres qui intervient rendent, en effet, indispensable une étude spécifique de chaque cas avant de décider du choix d'un dispositif de protection ou de renforcement (Panet, 1987). Cette étude doit aussi prendre en compte l'aspect esthétique et le coût du système de protection.

\section{1}

\section{Les différents types de renforcement}

On peut regrouper les dispositifs de protection et de renforcement en deux grandes classes.
Classe $A$ : parades actives

Type 1 : revêtement naturel (végétalisation, fascinage, implantation arbustive, reboisement de versant).

Type 2 : revêtement artificiel (béton projeté).

Type 3 : soutènement (contrefort, buton, ancrages). Classe B : parades passives

Type 1 : écran au niveau de l'emprise (écran grillagé blocs de béton, séparateur en béton, merlon de terre, surlargeur en pied de talus).

Type 2 : obstacle sur pente (mur barrage, chambre d'éboulis, écran de filets métallique).

Type 3 : contrôle des chutes de pierres sur le talus (couverture grillagée).

Type 4: ouvrage de transit (galerie paravalanche).

Type 5: abattage contrôlé (purge).

\section{2}

\section{Le renforcement des talus rocheux fissurés par ancrage}

Toute méthode de renforcement, qui tend à rétablir la continuité d'une masse rocheuse, permet d'améliorer grandement sa stabilité et de remédier à la rupture (Panet et al., 1976), c'est le cas des procédés de renforcement des massifs rocheux par des câbles ou des barres d'acier, qui se sont développés de plus en plus ces dernières dizaines d'années. On distingue deux techniques de renforcement par barres d'acier:

1) Le renforcement par ancrages actifs

Le renforcement des masses rocheuses par des tirants ou des barres précontraintes est une technique déjà ancienne. Des tirants de forte capacité (jusqu'à $10000 \mathrm{kN}$ ) ont été mis en œuvre en 1934, par André Coyne, au barrage des Cheurfas, en Algérie (Panet, 1987). Cette technique présente certains inconvénients tels que :

- le risque de rupture de la roche au niveau des zones d'ancrage où les contraintes sont relativement fortes ; - le risque de rupture à terme des câbles d'acier par corrosion (circulation d'eau le long de la discontinuité); - la diminution avec le temps par relaxation de la tension dans les câbles :

- le coût.

\section{2) Le renforcement par ancrages passifs}

Par renforcement passif, il faut entendre une armature qui est scellée au massif sur toute sa longueur et qui n'est pas mise en tension lors de sa mise en place (Azuar, 1977). S'il ne se produit pas de déformation dans le massif renforcé, les aciers passifs ne sont pas sollicités. Un des premiers exemples d'utilisation de cette technique est celui des appuis du barrage de Chaudanne en France (Panet, 1987).

Le fonctionnement des armatures passives est beaucoup plus difficile à analyser que celui des armatures précontraintes, car ce type de renforcement crée un milieu composite avec des interactions locales entre la roche et les armatures, notamment au niveau des intersections avec les surfaces de discontinuité. L'utilisation pratique des armatures passives a permis de mettre en évidence l'efficacité d'un tel procédé et l'expérience montre que le renforcement des massifs rocheux par ce type d'ancrage améliore grandement leur stabilité, 
c'est d'ailleurs maintenant un procédé couramment utilisé (Groupe français, 1977).

La plupart des méthodes de calcul des ancrages passifs utilisent des formules empiriques et des coefficients de sécurité élevés; elles se basent généralement sur l'une des deux hypothèses suivantes : soit la discontinuité est dilatante et on suppose que la barre est sollicitée en traction, soit la discontinuité est non dilatante et on suppose que la barre est sollicitée en cisaillement (Aydan et Kawamoto, 1987). En réalité, la barre travaille en même temps à la traction et au cisaillement (Bjurstrom, 1974; Lam, 1983; Ludvig 1983; Martin et Kaiser, 1984 ; Dight, 1985).

L'étude réalisée au Laboratoire central des ponts et chaussées de Paris (Asroun, 1993) a montré que le comportement à la rupture d'un ancrage passif est influencé par les principaux paramètres suivants :

a) Résistance à la compression de la roche $\left(\mathrm{R}_{\mathrm{e}}\right)$

$\mathrm{C}$ est la contribution de l'ancrage à la résistance de cisaillement de la discontinuité.

Selon la résistance à la compression simple de la roche $(R)$, on peut distinguer deux types de rupture d'un ancrage passif : une rupture par cisaillement pur dans le cas des roches dures $\left(\mathrm{R}_{\mathrm{f}}>100 \mathrm{MPa}\right)$ et une rupture par flexion avec formation de rotules dans le cas des roches tendres $\left(R_{c}<100 \mathrm{MPa}\right)$. En conclusion, on peut dire que pour des roches de résistance ( $R$ ) supérieure à environ $100 \mathrm{MPa}$, l'influence de $\mathrm{R}_{\text {sur la }}$ contribution de la barre (perpendiculaire à la surface de cisaillement) à la résistance au cisaillement est négligeable; la barre est cisaillée après un faible déplacement. Par contre,pour des roches tendres de résistance faible, la barre en écrasant le coulis, 1 roche se met dans une orientation qui la sollicite plus en traction qu'en cisaillement et, par conséquent, donne une contribution à la résistance au cisaillement la plus élevée (Fig, 1).



FG.1 L'influence de $R_{c}$ sur la contribution d'un ancrage passif.

Effect of $R_{\text {c }}$ on the contribution of a passive anchorage.

\section{b) L'inclinaison de la barre $(\theta)$}

On note $\theta$ l'angle que fait la barre avec la normale au plan de cisaillement.

La contribution de la barre à la rupture augmente notablement en passant d'un angle $\theta$ de $0^{\circ}$ à $30^{\circ}$ dans le cas des roches avec ( $\left.R_{c}=140 \mathrm{MPa}\right)$, mais n'augmente que très légèrement dans le cas des roches avec $R_{\text {f }}=$ $40 \mathrm{MPa}$ (Fig. 2). Cela peut s'expliquer par le fait que dans le cas d'une roche tendre, la barre (initialement perpendiculaire au plan de cisaillement) en écrasant le coulis et la roche prend une position favorisant la traction, avec une orientation $\theta$ avoisinant les $30^{\circ}$. Par contre, en passant d'un angle $\theta$ de $30^{\circ}$ à $45^{\circ}$, la variation de la contribution est presque nulle dans les deux cas de roche, la barre est bien orientée dans le sens du déplacement tangentiel, donc elle travaille essentiellement en traction et écrase très peu la roche et par conséquent a le même comportement dans les deux cas de roche : le type de roche ne joue pratiquement aucun rôle.

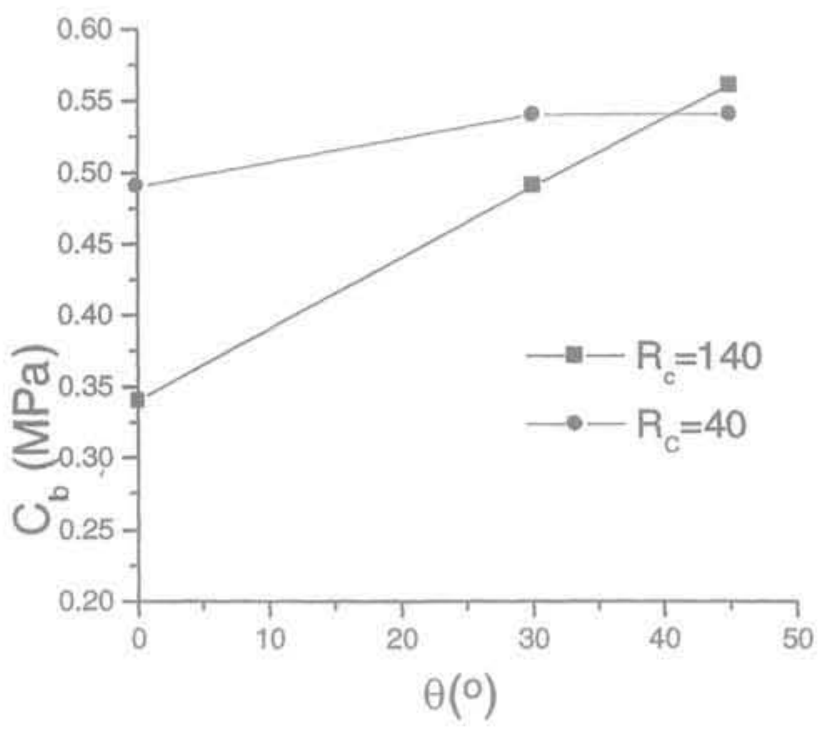

FG. 2 L'influence de $\theta$ sur la contribution d'un ancrage passif.

Effect of $\theta$ on the contribution of a passive anchorage. 
Asroun A. - Etude du comportement des ancrages passif's scellés au rocher. Thèse de doctorat, École centrale de Paris, 1993, $160 \mathrm{p}$.

Aydan O. Kawamoto T. - Toppling failure of discontinuous rock slopes and their stabilisation. $J$. of Min. Metall. Inst. of Japan 103, 1987, p. 763-770.

Azuar J.J. - Stabilisation des massifs rocheux fissurés par barres d'acier scellées. Rapport de recherche 73, Labora. toire central des ponts et chaussées Paris, 1977.

Bandis S., Lumsden A., Barton N. - Experimental studies of scale effect on the shear behaviour of rock joints. Int. J. Rock Mech. Min. Sci. Geomech. Abstr. 18, 1981, p. 1-21.
Benjelloun Z.H., Bertrand L., Feuga B. Etude du cornportement mécanique de la fracture rocheuse en cisaillement. Rock at Great Depth, Balkema, 1989, p. 433-439.

Bjurstrom S. - Shear strength of hard rock joints reinforced by grouted untensioned bolts. Proc. 3rd Int. Cong. on Rock Mechanics, ISRM, Denver, vol. II.B, 1974, p. $1194 \mathrm{FF}$

Dight P.M. - The theoretical behaviour of full contact bolts subject to shear and tension. Int. Symp. on the role of the rock mechanics, Zacatecas, Mexico, 1985. p. $290-297$.

Groupe français - Le renforcement des massifs rocheux par armatures passives. 4th Int. Cong, on Rock Mechanics, ISRM, Montreux, 1977.
Lam T.S.K. - Shear behaviour of concreteRock joints. Ph. D. Thesis, Monash University, Melbourne, Australia, 1983.

Ludvig B. - Shear tests on rock bolts. Proc. Int. Symp, on Rock Bolting, Abisko, 1983, p. 113-123.

Martin C.D. Kaiser P.K. - Analysis of rock slope with internal dilatation. Canadian Geotechnical Journal, 1984, p. 605-620.

Panet M. Rochet L., Azuar J.J. - Reinforcement of discontinuous rock masses by grouted untensioned bars. Séminaire de Jablona, Pologne, 1976.

Panet M. - Fenforcement des fondations et des talus à l'aide d'ancrages actifs et passifs. Proc. 6th Int. Cong. ISRM. Thema 2, Montréal, Generalbericht, 1987 